Revue d'histoire de l'Amérique française

FB. REVUE D.HISTOIRE DE L'AMÉRIQUE FRANÇAISE

\title{
Henri Bourassa et la guerre sud-africaine (suite et fin)
}

\section{James I. W. Corcoran}

Volume 19, numéro 3, décembre 1965

URI : https://id.erudit.org/iderudit/302492ar

DOI : https://doi.org/10.7202/302492ar

Aller au sommaire du numéro

Éditeur(s)

Institut d'histoire de l'Amérique française

ISSN

0035-2357 (imprimé)

1492-1383 (numérique)

Découvrir la revue

Citer cet article

Corcoran, J. I. W. (1965). Henri Bourassa et la guerre sud-africaine (suite et fin). Revue d'histoire de l'Amérique française, 19(3), 414-442.

https://doi.org/10.7202/302492ar d'utilisation que vous pouvez consulter en ligne.

https://apropos.erudit.org/fr/usagers/politique-dutilisation/ 


\section{HENRI BOURASSA ET LA GUERRE SUD.AFRICAINE * \\ (suite et fin)}

La Chambre des Communes connut, le 13 mars 1900, la première "journée Bourassa." Il prononça un discours de trois heures et demie, analysant complètement la guerre sud-africaine et la participation du Canada. Il en déduit que la véritable importance de l'envoi du contingent canadien consistait en cela qu'elle jette les bases d'une future fédération impériale. Cette conclusion, il la tire, non du simple fait de la participation, mais tout autant du mystère et des pressions dont elle a été entourée, et de sa connaissance des déclarations et des aspirations bien connues de Joseph Chamberlain. Il craint que les intérêts des colonies soient de plus en plus sacrifiés aux objectifs appelés "impériaux," mais qui ne sont rien d'autre que les plans mégalomanes d'hommes d'État anglais.

De plus, je sais que mon attitude n'est pas populaire, à l'heure actuelle, au moins. Mais ces inconvénients ne me touchent nullement. Plus se rapprochent de nous les résultats de la politique que je combats, plus je me sens prêt à subir le jugement loyal et impartial de mes concitoyens de toute origine; de ceux au moins qui me reconnaissent le droit d'être à la fois fidèle sujet britannique, Canadien avant tout et libéral constitutionnel. ${ }^{65}$

Bourassa défend ensuite sa loyauté mise en doute par plusieurs Canadiens.

Dois-je me laisser qualifier de sujet déloyal, de traître à mon parti parce que je refusai de suivre le gouvernement dans son évolution ? Je restai au point où mon chef, qui est celui du ministère, s'était placé dix jours auparavant, s'appuyant sur la loi

*Voir notre Revue, XVIII: 343-356; XIX : 84-105, 229-237.

65 Débats (1900), I : 1781-1782. 
et sur la constitution et sur les traditions les plus saines du parti libéral, britannique et canadien. ${ }^{66}$

Il cite nombre de journaux de langue française et de langue anglaise, libéraux et conservateurs, qui commentent favorablement sa position. En guise de réponse aux journaux de langue anglaise qui disent de lui qu'il n'est, après tout, qu'un Canadien français, et que l'on peut bien alors lui pardonner son ignorance du droit constitutionnel, il produit le témoignage du solliciteurgénéral "qui a admis que l'action du gouvernement, en termes techniques, ne se justifiait pas. Bien entendu, l'honorable gentilhomme a approuvé la politique du gouvernement pour des motifs sentimentaux et religieux." 67

Bourassa veut que l'on discute ouvertement le problème de la fédération impériale. La déclaration officielle du gouvernement canadien néglige complètement cette question, alors que la presse et le gouverneur-général continuent de donner à ce geste la portée d'un premier pas vers la fédération impériale.

Bourassa s'en prend ensuite à l'opinion publique sur laquelle Laurier s'est appuyé pour émettre l'ordre-en-conseil. Il énumère douze journaux qui, du moins partiellement, ont approuvé sa position. Selon lui l'opinion populaire n'est pas nécessairement bien renseignée et il présente un éloquent exemple de son propre rôle dans la vie politique en commentant les devoirs des députés.

Il est vrai que, sous un régime démocratique, nous sommes les serviteurs du peuple; mais le devoir nous incombe aussi de renseigner et d'instruire le peuple... Et si j'accuse le gouvernement, ce n'est pas tant d'avoir cédé de guerre lasse devant ce qu'il appelle l'opinion publique, mais parce qu'il a négligé d'éclairer cette opinion par ses organes et ses partisans. ${ }^{68}$

Il discute de nouveau le rôle du parlement canadien; il le replace dans la conjoncture des plans impérialistes.

${ }^{68}$ Débats (1900), I: 1786.

${ }^{67}$ Débats (1900), I: 1786.

68 Débats (1900), I: 1797. 
Le parlement est fait pour la discussion; c'est l'endroit où l'on peut décider des grandes questions publiques sous la garantie réelle de l'ordre et de la liberté... La fidélité à la Reine ne veut pas dire fidélité à Gladstone ou à Salisbury, ou à Chamberlain ... Nos ministres doivent prendre leurs instructions non des ministres de Westminster, mais du parlement du Canada, dont ils sont un comité et auquel ils sont exclusivement responsables. ${ }^{69}$

La loyauté à la reine est l'une des restrictions importantes soulevées par Bourassa. Par sa réponse à l'ordre-en-conseil, Chamberlain a voulu lier loyauté à la couronne et loyauté au gouvernement. Sans esquisser de système de rechange pour l'Empire, l'orateur soutient que l'état de colonie autonome dissocie la loyauté canadienne envers la couronne de tout lien avec les fluctuations de la vie politique anglaise. L'on peut faire remonter à sa lettre de démission cette théorie implicite du "Commonwealth". Bourassa est au surplus d'avis qu'il ne faut pas imputer à l'opinion publique mais à la lettre de M. Chamberlain la décision de Laurier au sujet de l'envoi de contingents.

... on envoie alors au governement canadien une lettre qu'on a fait publier au préalable dans les journaux de Londres, acceptant des offres qui n'ont jamais été faites; et l'on fixe une date d'envoi assez rapprochée [pour le départ des troupes] pour ne pas permettre de convoquer le parlement. On ne laisse pas même le temps d'une saine réflexion; l'on place le gouvernement en cette alternative: obéissance immédiate et complète, ou refus c'est-àdire accusation de déloyauté et de haute trahison répandue à l'envie par tout l'empire. ${ }^{70}$

Les événements qui ont amené Laurier à échanger sa politique révèlent clairement les pressions exercées et la stratégie employée, et cela sans aucune discussion ouverte sur le sujet de l'impérialisme. Lorsque Bourassa déclare que le précédent c'est le fait accompli, il semble bien que nous soyons en présence d'une analyse véritable de l'intention du secrétaire d'Etat aux

${ }^{69}$ Débats (1900), I: 1800.

70 Débats (1900), I: 1801. 
Colonies. Les gestes de Chamberlain rappellent cette maxime de son contemporain, Theodore Roosevelt: "Parlez à voix basse et armez-vous d'un bon bâton." Bourassa le faisait d'ailleurs observer: l'on n'avait accepté aucune offre de contribution de guerre venant d'une colonie de la couronne. C'est que semblables contributions pouvaient être obtenues à n'importe quel moment. Ce que l'on voulait, c'étaient les contributions publiques et officielles des colonies autonomes.

Minto pressa Laurier de reconsidérer la question d'un contingent officiel, anticipant ainsi sur la lettre de Chamberlain du 4 octobre qui informait Minto: "Nous n'avons l'intention d'accepter aucune offre de volontaires. Nous n'avons pas besoin des hommes et la raison même de l'offre disparaîtrait si elle n'était pas faite par le gouvernement de la colonie."71

Suit l'histoire des discussions au parlement australien. Bien que les journaux canadiens aient affirmé le contraire, un fort mouvement d'opposition s'était dessiné dans chacune des colonies australiennes; dans chacune d'elles, la question alla au parlement. En Australie du Sud, le seul vote de l'Orateur, à la Chambre Haute, permit le passage de la résolution autorisant l'envoi de troupes. Par là Bourassa essayait d'endiguer le flot de demi-vérités, d'appels aux sentiments et à l'unité de l'empire. $\mathrm{Au}$ moment de la publication de l'ordre-en-conseil, quels motifs a-t-on allégués pour ne pas réunir le parlement? Les députés ne devraient pas oublier le danger de laisser le cabinet prendre des décisions aussi cruciales sans l'assentiment de la Chambre.

Mais on devrait éviter avec soin de tendre à l'excès contraire et laisser le cabinet prendre à la fois la place de la couronne et du parlement. Ne permettons pas à nos ministres d'oublier qu'ils ne sont qu'un comité exécutif du parlement. Qu'ils se gardent d'escompter trop largement l'obéissance servile d'une majorité de partisans et d'adopter par décrets de cabinet des mesures de la plus haute importance qui ne devraient voir le jour et ne s'exécuter qu'avec le concours du parlement! 72

71 Wade, The French Canadians, 521.

72 Débats (1900), I: 1789. 
Sans doute, le parlement canadien aurait cédé aux pressions et aux appels des impérialistes; mais il semble certain qu'un débat sur les questions en jeu aurait suscité une considérable opposition à l'envoi de troupes. Tel avait été le cas dans les autres colonies autonomes. Et cet envoi de troupes, et cette urgence prétendue et déployée pour les enrôler et les embarquer!

Inutile de répéter tous les arguments qu'on a fait valoir ici même, établissant que nos troupes n'étaient point requises dans l'Afrique australe. Les autorités britanniques ne comptaient que sur ces cinq cents soldats canadiens. Qui prétendra que le sort des armes anglaises reposait sur cinq cents hommes? Qui osera affirmer qu'aujourd'hui même, la présence ou l'absence de nos deux mille soldats peut modifier le cours des évènements? [sic] ${ }^{73}$

Nous avons déjà lu la lettre de Chamberlain à Minto où il dit ne pas porter d'intérêt aux hommes eux-mêmes. A propos de l'urgence du départ de ces troupes, Bourassa l'impute à Chamberlain lui-même.

La seule indication d'urgence que je puisse retracer se trouve dans la dépêche de $M$. Chamberlain, en date du 3 octobre, reçue ici quelques jours plus tard... Cette dépêche demandait que les troupes fussent expédiées avant le 31 octobre. Il y avait alors, et il y eut encore pendant plusieurs mois, des milliers et des milliers de régiments anglais cantonnés en Angleterre. Je ne puis accepter la théorie que les désirs ou les ordres de M. Chamberlain suffisent à constituer un plaidoyer d'urgence que les faits contredisent. Notre gouvernement n'aurait-il $\mathrm{pu}$, à l'exemple de M. Lyne, premier ministre des Nouvelles Galles du Sud, expliquer à M. Chamberlain que nous vivons sous un régime constitutionnel et que les troupes seraient expédiées dès que le parlement aurait décrété leur enrôlement?

Mais M. Chamberlain [a] ... disposé plus nettement encore de ce prétexte de besoin urgent ... [en proclamant] que ce déploiement fastueux de militarisme impérial n'était pas organisé pour les besoins

${ }^{73}$ Débats (1900), I: 1789-1790. 
de cette guerre; mais qu'on voulait par là donner au monde un exemple et un avertissement. J'admets volontiers que cette idée n'est pas dépourvue de grandeur; mais n'aurait-on pu donner cet exemple et cet avertissement un mois plus tard? Quant à nous, la leçon n'aurait-elle pas été aussi frappante et aussi profitable, si le parlement l'avait donnée lui-même au lieu de la laisser signifier par un simple décret ministériel? 74

Et venons-en à la prétention de l'absence de précédent. Bourassa fait lecture d'une série de citations extraites de journaux anglais, français et étrangers: leur interprétation du geste du gouvernement canadien corroborait la sienne. D'où procède cependant la réelle grandeur de l'empire britannique?

Qu'est-ce qui a fait l'Angleterre ce qu'elle est? Qu'est-ce qui lui a attaché toutes ses grandes colonies, sinon une politique de paix, d'industrie, de commerce, de décentralisation complète, militaire et civile, une politique où elle laissait à chaque colonie le droit et le pouvoir de développer ses propres ressources suivant sa situation particulière? Pour ma part, j'ai encore foi dans les hommes et dans la politique qui ont fait de l'Angleterre la terre classique de la paix et de la liberté. Ces hommes occuperont un rang plus élevé dans les annales de l'histoire que les dominateurs bruyants et ambitieux qui veulent faire participer l'Angleterre aux rapines territoriales auxquelles se livrent les autres nations! ${ }^{75}$

C'est là la description normale des relations qui doivent exister entre le Canada et la Grande-Bretagne. D'où l'opposition de Bourassa à tout changement impulsif et irraisonné de cette situation.

On m'a demandé ce que je voulais. La réponse est courte et directe. Je veux qu'on respecte la constitution de mon pays telle qu'elle est, et je dis à ceux qui n'en sont point satisfaits: si vous voulez la modifier, dites franchement ce que vous voulez. Ne

74 Débats (1900), I: 1790-1791.

75 Débats (1900), I: 1811. 
venez pas au milieu d'une tempête populaire chercher à mettre le feu au temple.

M. Chamberlain et ses disciples fanatiques et ses apôtres inconscients nous conduisent à une révolution constitutionnelle dont personne ne peut prévoir les conséquences.

Mes objections à tout changement dans nos relations avec la Grande-Bretagne ne sont ni aveugles ni déraisonnables. Je ne demande qu'à me laisser convaincre. Mais j'appuie sur un point: Nous devons savoir exactement où nous sommes et où l'on nous conduit. Avant qu'une évolution ne s'opère, on doit établir nettement ce qu'on veut nous faire abandonner, et ce qu'on nous offre en retour. Et le parlement lui-même - et, à plus forte raison, son comité exécutif - n'a pas le droit d'engager l'avenir de ce pays sans connaître à fond le sentiment vêritable du peuple.

Qu'on soumette au pariement des propósitions clàires et nettes: qu'elles soient discutées à fond et qu'un plébiscite dégageant la question de tous les autres débats politiques, la règle définitivement. ${ }^{76}$

Ce passage revêt un intérêt spécial. Il illustre la position de Bourassa sur l'envoi de troupes au Transvaal. Il avait démissionné et s'était présenté devant ses électeurs en se réclamant de ce qu'il croyait être un exceptionnel problème constitutionnel. Nous lui devons d'avoir fourni au parlement une compréhension claire des conséquences impériales de l'envoi des troupes. Bourassa saisit l'occasion pour décrire l'origine de ses convictions politiques.

Je suis un libéral de l'école anglaise. Je suis un disciple de Burke, de Fox, de Bright, de Gladstone et des autres "Little Englanders," qui ont fait l'Angleterre et ses possessions ce qu'elles sont aujourd'hui, et je ne déserterai pas les rangs de leurs disciples fidèles, parce qu'il plairait à M. Chamberlain et à d'autres radicaux renégats, dévorés du délire de l'ambition, de traiter ces grands hommes d'insensés. On peut dire que Gladstone a changé d'opinion sur plusieurs questions. C'est vrai. Mais

76 Débats (1900), I: 1811-1812. 
- et c'est là la marque de son génie et de son libéralisme - toutes ses variations l'entraînaient du plus étroit au plus grand. Qu'il s'agit de questions d'Eglise, d'affaires d'Etat ou de science sociale, tous ses changements d'opinion ont été une marche constante dans la voie du libéralisme.

Je suis né libéral, je mourrai libéral. Et libéral je resterai, même à travers les flots du torysme qui peuvent submerger un instant les champs du libéralisme. Et ni roi, ni gouverneur, ni ministre, même dans mon propre parti, et nulle foule aveugle ne peuvent m'imposer des convictions qui ne sont pas miennes. ${ }^{77}$

Preuve on ne peut plus éloquente de l'individualisme profond de Bourassa; preuve aussi de sa compréhension de l'évolution politique de Gladstone. Bourassa, nous l'avons déjà signalé, faisait passer le principe avant le parti. Il conclut son discours en présentant sa motion.

Il est une autre cause qui fait que ma proposition est non seulement utile, mais absolument nécessaire. Cette cause provient des faits graves que j'ai soumis à la Chambre. La presse reptile tout entière, ainsi qu'une portion notable des journaux respectables libéraux, conservateurs, et indépendants - en Angleterre et au Canada, et je puis ajouter toute la presse étrangère, n'ont tenu aucun compte de la réserve faite par le gouvernement dans son décret du 13 octobre. Tous ces journaux n'exprimèrent pas les mêmes opinions sur cette question, les uns approuvant, les autres condamnant l'attitude du cabinet; mais ils ont été unanimes à interpréter l'action ministérielle comme je l'ai fait moi-même, et à dire que le fait accompli crée le précédent.

Mais ce qui est encore plus grave et donne une force nouvelle à ma prétention, c'est que le gouvernement britannique, par l'entremise du secrétaire colonial, à la demande de qui nous agissons, a donné à l'acte du ministère la même interprétation. Il a accepté l'envoi de nos soldats, comme une preuve de notre désir de voir la chose se renouveler; il a

77 Débats (1900), I: 1821. 
méconnu entièrement la réserve faite par le gouvernement canadien.

Je ne suis pas entré dans cette voie pour acquérir du renom ou de la popularité. Je n'ai qu'un but: c'est de faire reconnaître et proclamer par la Chambre les principes par excellence du gouvernement représentatif.

Tout ce que je demande à la Chambre, en somme, c'est de déclarer que le Canada est un pays libre, prêt d'ailleurs à écouter des propositions acceptables si la Grande-Bretagne nous dit qu'elle a besoin de notre aide. Mais, s'il s'agit de donner à ce pays une nouvelle orientation politique, je refuse au secrétaire colonial, et à tout membre du cabinet britannique, et à tout représentant du gouvernement impérial au Canada le droit de dicter à un peuple libre quelle sera cette politique nouvelle. ${ }^{78}$

Je me sentirais amplement récompensé, si je pouvais faire affirmer par le parlement canadien, que le vieil esprit britannique, source de notre autonomie et de nos libertés anime encore mon pays.

Je propose donc que tous les mots après "que", soient retranchés et remplacés par les suivants:

Que cette Chambre insiste sur le principe de la souveraineté et de l'indépendance du parlement comme étant la base des institutions britanniques et la sauvegarde des libertés civiles et politiques des citoyens britanniques, et refuse, en conséquence, de considérer l'action du gouvernement au sujet de la guerre de l'Afrique du Sud comme un précédent qui doive engager ce pays dans toute action à venir.

Que cette Chambre déclare, de plus, qu'elle s'oppose à tout changement dans les relations politiques et militaires qui existent actuellement entre le Canada et la Grande-Bretagne à moins que tel changement ne soit décrété par la volonté souveraine du parlement et sanctionné par le peuple du Canada. ${ }^{79}$

78 Débats (1900), I: 1827-1828.

79 Débats (1900), I: 1829. 
La principale préoccupation de Bourassa est d'affirmer la suprématie du parlement canadien et d'en obtenir l'assentiment dans le domaine des relations entre la Grande-Bretagne et le Canada. Même si Bourassa constatait que l'insertion quant à "l'absence de précédent" dans l'ordre en conseil était lettre morte pour le secrétaire d'Etat aux colonies, l'action du parlement du Canada, estimait-il, pouvait rendre cette clause politiquement opératoire. Le fait accompli continuerait, certes, d'exister, mais la solennelle déclaration du gouvernement canadien pourrait catalyser l'impérialisme et le jingoïsme de l'époque. Le ton modéré de la résolution est remarquable. Laurier avait pu agir sans le parlement en octobre et ce dernier ne l'avait pas censuré, tellement étaient forts les sentiments impérialistes qui agitaient le peuple canadien. A quelques exceptions près, parmi les députés de langue française, le parti conservateur avait ouvertement préconisé une politique de participation plus directe et plus complète: le parti libéral était également aux prises avec la fièvre impérialiste. Les députés canadiens-français, même opposés à la participation, estimaient que leur loyauté première allait à leur chef, à Laurier, le premier Canadien français à devenir premier ministre du Canada. Les conservateurs au pouvoir, ils le savaient, engageraient plus d'hommes et plus d'argent au service de la cause impériale. Laurier pouvait à ce moment compter sur l'appui et la sympathie du Canada français tout en se croyant tenu de faire des concessions à l'impérialisme anglo-saxon. Le premier ministre se leva pour répondre au discours de Bourassa.

Je n'ai rien à dire des principes énoncés dans la proposition du député de Labelle; je ne suis pas prêt, cependant, à en accepter l'entière phraséologie, pas plus que je suis prêt à admettre que le moment a été bien choisi pour la présenter au parlement... Si l'honorable député ou tout autre de ses collègues, proposait sous forme d'amendement au vote des subsides, que deux et deux font quatre ou qu'il y a douze mois dans l'année, ou qu'il est du devoir d'un bon chrétien d'observer les dix commandements, personne ne s'y objecterait; mais, d'un autre côté, personne ne se croirait tenu de faire 
consigner cette proposition dans les archives du parlement. ${ }^{80}$

Laurier fit cas des dépenses, non des troupes, et déclara que la constitution n'avait pas été violée. Il conclut en niant qu'on lui ait forcé la main.

Non, l'Angleterre ne nous a pas forcé à agir; ni M. Chamberlain ni Downing Street ne nous ont forcés à faire ce que nous avons fait... Nous avons exercé la pleine indépendance de notre pouvoir souverain. Ce que nous avons fait, nous l'avons fait de notre plein gré... ${ }^{81}$

La motion fut défaite par 119 voix contre 10. Fait inquiétant: les dix opposants portaient des noms à consonance française. Bourassa continua de s'opposer à la politique sud-africaine du gouvernement. Le 4 juin 1900, il souleva un sujet de débat qu'il devait développer lors de son second grand discours et de sa seconde motion sur les affaires sud-africaines, durant la session de 1901.

Le gouvernement, ou quelqu'un de ses membres, a-t-il été consulté sur les conditions les plus propres à faire cesser la guerre dans le Sud-africain? Le gouvernement se propose-t-il de suggérer quelque moyen ou d'exprimer son opinion à ce sujet? ${ }^{82}$

Le Premier ministre répondit:

Ni le gouvernement ni aucun de ses membres n'ont été consultés sur les conditions les plus propres à faire cesser la guerre dans le Sud-africain. Le gouvernement ne se propose pas de suggérer quelque moyen ou d'exprimer son opinion à ce sujet..3

Le 7 juin 1900, Bourassa s'élève contre une adresse de félicitations à la reine au sujet de la chute de Prétoria et de la conduite de la guerre.

80 Débats (1900), I: 1830-1831.

81 Wade, The French Canadians, 483-84.

82 Débats (1900), II : 6658.

83 Débats (1900), II : 6658. 
Le courage des soldats, la bravoure des officiers ne dépendent pas des causes de cette guerre; aussi je me joins de tout cœur au premier ministre et à l'honorable député pour féliciter les vaillants soldats qui ont parcouru les champs de bataille de l'Afrique du sud. Mais lorsqu'il s'agit de se réjouir des résultats de la guerre et de féliciter Sa Majesté et son gouvernement sur les causes du conflit, sur la politique qui a entraîné l'Angleterre et l'empire dans cette lutte sanglante, je me sépare d'eux. Car ces causes sont injustes à mes yeux et le succès ne les a point légitimées. ${ }^{84}$

Je ne puis approuver une politique qui [renie] les meilleures traditions de l'Angleterre ... [qui] ébranlera aussi tout l'empire britannique en y répandant cet esprit de jingoïsme et d'orgueil, qui n'est certes pas l'indice de la force et de la générosité. ${ }^{85}$

Ce dernier passage renferme la première allusion indirecte à ce que Bourassa devait plus tard longuement développer, diagnostiquant dès lors la décadence de l'empire. L'accueil fait à son discours, en chambre, illustre bien le jingoïsme et l'orgueil qu'il dénoncait. Les députés se levèrent et, à l'unisson, poussèrent trois hourrahs pour Sa Majesté et conclurent avec le "God Save the Queen". Le 5 juillet 1900 - la session tirait à sa fin - Bourassa prit encore la parole au sujet, cette fois, de la nourriture supplémentaire que le gouvernement devait fournir aux troupes canadiennes en Afrique du Sud.

Monsieur le président, il est très grave, à mon avis, d'apprendre à la fin de juin que nos troupes souffraient en Afrique du Sud; qu'elles souffraient à cause de l'insuffisance des vivres fournies par les autorités impériales; et que le ministre de la milice eût dû attendre la demande d'enquête en cette chambre avant de prendre des dispositions pour que les soldats canadiens soient convenablement traités par les autorités impériales.

Monsieur le président, voilà qui prouve qu'une action suit l'autre. A l'offre du premier contingent, on nous a dit que le gouvernement ne faisait en

${ }^{84}$ Débats (1900), II: 6936-6937.

85 Débats (1900), II: 6938. 
somme que permettre à des volontaires d'aller se battre en Afrique. On nous a dit que nous leur donnions des vaisseaux et que nous les nourrissions jusqu'au point d'arrivée mais que, dès ce moment, ils devenaient soldats britanniques, incorporés, à l'armée britannique, payés et nourris par le gouvernement britannique. Une action suit l'autre. $\mathrm{Au}$ moment de l'envoi du second contingent, il n'était plus question de donner le choix à ceux qui voulaient combattre; nous leur avons donné des chevaux, des fusils, des selles, nous avons ajouté un excédent de solde. Et maintenant l'on nous demande d'approuver un rapport qui décrète que le Ministre de l'Approvisionnement a bien fait en leur donnant un supplément de nourriture. ${ }^{86}$

C'était encore demander à la Chambre d'approuver un fait accompli. Un mince vote de 66 à 52 ratifia le geste du gouvernement.

Le Parlement s'ajourne bientôt, le 17 juillet. Des élections générales auraient lieu à la mi-octobre. La rumeur veut qu'Henri Bourassa tente de fonder un tiers parti avec un programme anti-impérialiste. Par la force des choses, ce parti serait canadien-français. Le 30 septembre 1900, en ouvrant sa campagne à Papineauville, Bourassa déclare qu'il continuera d'appuyer la politique générale du parti libéral, sauf en ce qui a trait à la guerre sud-africaine. Il reçoit l'investiture officielle du parti libéral mais, pas plus qu'en 1896, il ne veut accepter de fonds électoraux. Au cours de la campagne, cependant, le parti libéral dépense $\$ 500.00$ dans le comté de Labelle, à l'insu de Bourassa. Lorsqu'il découvre ce fait, il insiste pour rembourser cette somme à la caisse du parti, menaçant de dévoiler l'usage que l'on fait de cet argent. Le remboursement est accepté et Bourassa maintient son indépendance à l'intérieur de son parti. Bourassa avait écrit à Laurier, après la dissolution du parlement:

Vous n'aurez même pas la consolation que vous désirez si sincèrement, celle d'être un pacificateur

86 Débats (1900), III : 9328-29. 
dans votre propre pays. La paix, quelquefois, est pire que la guerre.

Il me semble que deux extrêmes doivent être évités: l'opposition irraisonnée à la volonté populaire sous le prétexte qu'elle fait violence à la vérité; la servilité vis-à-vis la volonté populaire sans souci du droit et de la justice. La postérité sera sévère pour vous. ${ }^{87}$

La réponse de Laurier, alors en campagne électorale, illustre le contraste psychologique qui oppose ces deux hommes. Laurier se considère comme l'agent d'une union forte et durable entre les deux groupes ethniques du Canada. Il lui semble nécessaire de faire des concessions à l'impérialisme des Canadiens anglais pour préserver un parti libéral uni et bi-racial. Une attaque contre l'impérialisme, venant d'un Canadien français, suscite aussitôt la suspicion générale nonobstant son mérite. Laurier répond à Bourassa:

Il se peut que j'échoue dans le rôle de pacificateur que je me suis tracé, mais il demeure néanmoins vrai qu'il n'y a aucune autre politique qui convienne actuellement à notre pays; et si, au lieu de suivre ma politique, je m'étais conformé à vos vues, le résultat aurait tout simplement été désastreux; si je puis m'exprimer en toute franchise, je suis surpris qu'un homme aussi logique que vous l'êtes ne voyiez pas cette vérité évidente. ${ }^{88}$

"Logique", mot intéressant qui indique clairement ce qui sépare ces deux hommes sur la question de la guerre. Laurier parle de la logique de la politique. Le désastre, pour lui, aurait été un désastre électoral autant que, ou peut-être à cause de l'explicite question de race qui en aurait été l'enjeu: Bourassa, lui, faisait fi de la logique politique pour traiter le problème en termes de faits et de principes. Il n'était pas raciste. Il avait rejeté l'occasion de fonder un parti qui, par la force des choses, se serait limité "de facto" à une race: ceci s'explique en partie

${ }^{87}$ Lettre de Henri Bourassa, le 14 août 1900, à Sir Wilfrid Laurier (Papiers Bourassa).

88 Lettre de Sir Wilfrid Laurier, le 20 août 1900, à Henri Bourassa (Papiers Bourassa). 
par le fait que Bourassa était doué d'un tempérament d'orateur ou, comme le suggère le chanoine Groulx, qu'il était un propagandiste d'idées. ${ }^{89}$ Les données libérales sur lesquelles il fondait son nationalisme canadien sont en elles-mêmes incompatibles avec une approche raciste de la politique. Bourassa est réélu aux élections générales. Il continue son opposition à la politique de guerre du gouvernement, opposition qui atteignit son point culminant le 12 mars 1901. Dès le début du discours qu'il prononce devant le parlement, Bourassa réfute les accusations selon lesquelles sa position était essentiellement raciste.

J'ai toujours eu si peu l'intention de soulever l'agitation raciale sur cette question, cette idée a toujours été tellement absente de mes déclarations que je n'ai jamais pris la peine de réfuter cette accusation. ${ }^{90}$

Bourassa arrive rapidement à l'argüment-principe de sôn discours et il l'incorpore à sa motion.

L'on me dit que le Canada n'a pas le droit de dire que l'on devrait mettre fin à cette guerre où notre contribution requise de 500 hommes a été élevée à plus de 3,000 hommes, où une dépense nationale de quelques milliers de dollars est rendue à plus de deux millions. ${ }^{21}$

Et voici l'orateur qui en revient au thème de la décadence britannique.

L'on a dit, imprimé, et chanté sur tous les tons, depuis plus d'un an que cette guerre a fait passer les colonies britanniques autonomes au rang de nations libres alliées à la Grande-Bretagne...

Ces effusions triomphantes d'orgueil colonial me rappellent immanquablement les années de décadence de l'Empire romain: poètes et rhéteurs, oublieux de la vie rude mais libre de leurs ancêtres, exaltaient la gloire de César et vénéraient ses images

$89 \mathrm{Au}$ cours d'une entrevue avec le chanoine Groulx, à Montréal, celui-ci décrivit Bourassa comme "essentiellement [sic] propagandiste d'idées [sic].

90 Débats (1901), I: 1290-91.

91 Débats (1901), I: 1291. 
en or, parce qu'on les faisait participer à ses débauches raffinées; ... Rome possédait un empire plus vaste, ses provinces étaient plus soumises, elle vantait plus sa force à la veille de sa chute qu'à n'importe quel autre moment de son histoire. Mais nous savons maintenant, et ses citoyens clairvoyants le savaient alors, que l'heure de sa plus grande force morale et matérielle fut celle où ses hommes d'Etat pensaient plus à corriger les abus domestiques et à conserver le réel esprit romain de liberté qu'à piller l'univers et vénérer la force brutale et l'avarice insatiable dans la personne de l'Empereur. ${ }^{22}$

Il était puéril d'affirmer que les nations autonomes devenaient des nations libres en participant à la guerre des Boers. Effectivement, on s'était éloigné du statut de nation. ${ }^{93} \mathrm{Au}$ surplus Bourassa partageait, avec les libéraux de tout l'empire, le sentiment que le militarisme impérial et le jingoïsme présageaient la décadence de l'Angleterre. C'en était fini de son époque de grandeur d'empire libéral. Il n'insista pas trop sur ce point, plus propre à susciter la colère que la compréhension des Canadiens anglais. Notons cependant qu'il exprime une protestation qu'élevaient des libéraux anglais. Preuve ce texte non équivoque:

La suprématie de cette grande race a été établie dans des circonstances modérément favorables, mais c'est avec générosité que sont arrivés tous les maux qui sont associés, dans l'histoire, à la chute de tous les Empires et de toutes les Nations...

Le cancer de l'argent a dévoré la société britannique. L'arbre est pourri, du faîte aux racines. L'homme le plus immoral joue, devant le public, le rôle du plus grand philanthrope...

Voilà le danger qui menace la race anglo-saxonne. Ce ne sont pas les mers courroucées des nations latines ou autres qui menacent la race anglo-saxonne, mais ce sont le ver cancereux de son propre cœur, la paresse, l'indolence, l'immoralité et la luxure, le

92 Débats (1901), I: 1292.

93 (Citation extraite d'un article de Lord Charles Beresford dans le North American Review, décembre 1900 (LXXI : 806-807), Bourassa, Great Britain and Canada, 1902 (appendix), cxxviii. 
manque de virilité, de chevalerie, de courage moral et de bravoure, que son sein nourrit.

Bourassa reprend:

Je vais simplement rappeler aux membres de cette chambre, en particulier à ceux qui s'enorgueillissent de notre position dans l'Empire qu'il est de la plus grande urgence de nous faire respecter, non seulement sur les champs de bataille mais dans les conseils de Sa Majesté, si nous ne voulons pas que le gouvernement britannique nous considère seulement comme un terrain colonial de spéculation profitable. ${ }^{94}$

Et voici une liste complète de tous les gestes irréguliers de Chamberlain à l'égard du Canada dans cette guerre.

J'ai décrit en chambre, à la dernière session, la procédure singulière suivie par le secrétaire d'Etat aux colonies: l'usage qu'il fit de notre résolution du Transvaal de 1899 pour dire que nous favorisions sa politique arrogante et provocatrice; l'acceptation de nos offres d'assistance avant qu'elles n'eussent été faites; la publication par la presse londonienne d'une dépêche officielle adressée à Lord Minto, et ce avant qu'elle soit parvenue à destination; et, surtout, sa réplique insolente au décret du 13 octobre. Depuis lors, nous avons eu une autre preuve de l'audace grandissante du maître de l'empire. ${ }^{95}$

Cette preuve, ce sont les documents parlementaires du 4 juin 1900:

Le gouvernement, ou quelqu'un de ses membres, a-t-il été consulté sur les conditions les plus propres à faire cesser la guerre dans le Sud-africain? Le gouvernement se propose-t-il de suggérer quelque moyen ou d'exprimer son opinion à ce sujet? ${ }^{96}$

Laurier réplique:

Ni le gouvernement ni aucun de ses membres n'ont été consultés sur les conditions les plus propres à

94 Débats (1901), I: 1293.

95 Ibid.

96 Débats (1901), I: 1293. 
faire cesser la guerre dans le Sud-africain. Le gouvernement ne se propose pas de suggérer quelque moyen ou d'exprimer son opinion à ce sujet. ${ }^{97}$

\section{Bourassa continue:}

Durant la présente session, le 18 février, j'ai posé la question suivante: Le gouvernement canadien, ou quelqu'un de ses membres, a-t-il été consulté par le gouvernement anglais, sur les affaires sud-africaines, depuis le 1er juin dernier?

Est-ce que le gouvernement canadien, ou quelqu'un de ses membres, a donné quelque avis ou présenté quelque suggestion au gouvernement britannique sur cette question?

[Le premier ministre] "Le gouvernement canadien, pas plus qu'aucun de ses membres, n'a été consulté par le gouvernement anglais, sur les affaires sudafricaines, depuis le 1er juin dernier. Aucun membre du gouvernement canadien n'a donné quelque avis sur cette question."

Traversons maintenant l'océan, allons au palais de Westminster, la mère des parlements, source et gardienne de la liberté britannique, de la justice britannique, de la vérité britannique. Dans le rapport parlementaire du Time, le 7 août dernier, voici ce que je lis.

“M. Faber (York) a demandé au secrétaire d'Etat aux colonies si, à la lumière de la participation canadienne et australienne au conflit sud-africain, l'on se proposait de demander l'opinion des gouvernements de ces pays sur le règlement du différend et le mode de gouvernement du Transvaal et de l'Etat d'Orange après la guerre."

Ayant maintenant à l'esprit les déclarations du premier ministre du Canada du 4 juin 1900 et du 18 février 1901, prêtons l'oreille à la réponse de l'oracle du nouvel univers britannique:

[M. Chamberlain] "Je connais déjà les vues des colonies du Canada et de l'Australie sur les principaux aspects du futur règlement, et le gouvernement de Sa Majesté est en accord complet avec eux sur la nécessité de l'annexion, de l'établissement d'un 
gouvernement appuyé par l'armée, et de l'ultime espoir d'un gouvernement autonome et représentatif pour ces deux colonies ..."

Voilà qui me justifie, je crois, d'en appeler à cette chambre, au nom du respect que se doit le Canada, pour mettre fin à ce traitement arbitraire. Je fais appel à ceux qui, au moins, n'ont pas atteint un tel degré de dévotion pour M. Chamberlain que se constituer un instrument de sa main soit un honneur pour le Canada ... et je puis entrevoir le jour où le jugement du peuple canadien, anglais comme français, ne sera pas aussi sévère envers moi que l'ont été les discours, les votes, les chants, et les hurlements qui ont marqué les débats de la dernière session. ${ }^{98}$

Pour Bourassa le problème en est un d'orgueil national et de respect personnel face aux accrocs dissimulés et malvenus faits aux prérogatives du Canada. Et l'impérialisme, quel visage lui a-t-on fait?

On n'a présenté l'impérialisme, au Canada, ni d'une façon identique ni d'une façon honnête. Dans les provinces anglaises, on l'a limité aux sentiments que l'on a fait valoir dans cette chambre, chaque parti réclamant la première place dans ce combat de dévotion envers la mère-patrie; mais on n'a discuté ni les aspects constitutionnels ni les ultimes conséquences de notre participation militaire.

Dans la province de Québec, lorsque l'on a soulevé le problème, les deux partis se sont mutuellement accusés d'avoir déployé un étalage de jingoïsme qui avait forcé la main du gouvernement. Un acte de vertu dans un coin du pays devenait donc un crime dans l'autre coin.99

Et je me permets de dire, en passant, à tous ceux qui veulent sincèrement la paix et l'harmonie entre

98 Débats (1901), I: 1294-95.

09 Les victoires britanniques en Afrique avaient diminué la tension au Canada, mais les conservateurs faisaient encore appel aux sentiments raciaux. Wade, The French Canadians, 485. Au Québec, les libéraux pressaient les Canadiens français de se ranger derrière un premier ministre français et catholique contre les fanatiques de l'Ontario, tandis que sir Charles Tupper s'efforçait de prouver, au nom des conservateurs, que Laurier était "trop anglais pour lui ... Wade, The French Canadians, 486. 
les deux races qui forment notre pays, que c'est en vain qu'ils essayeront d'obtenir cet heureux résultat s'ils continuent de traiter de double façon les grands problèmes du pays. ${ }^{100}$

Un panégyrique du libéralisme anglais va conclure ce discours:

Il se peut que j'aie blessé les sentiments des ministériels en montrant que le double jeu mené tout au long de cette crise est un manque de fermeté qui blesse profondément tous ceux qui sont venus au vieux parti libéral à cause de son courage moral, de son attachement désintéressé aux principes, et de son heureux penchant à préférer la vérité au succès. ${ }^{101} \mathrm{M}$. le président, je m'enorgueillis, et légitimement, de ce que la position que j'ai prise sur ce problème s'appuie fermement sur le respect de la constitution et sur une dévotion réelle aux intérêts canadiens de préférence aux visées politiques de M. Chamberlain. Ce faisant je suis toujours resté fidèle aux véritables traditions du libéralisme anglais. ${ }^{102}$

Voici la résolution de l'orateur:

Que, tout en adhérant fermement aux principes fondamentaux de l'autonomie canadienne, et tout en refusant d'admettre que l'intervention du Canada en Afrique du Sud ait engagé ce pays à toute future participation aux guerres et politiques de la GrandeBretagne...

Cette chambre est d'avis que la contribution canadienne au conflit sud-africain, contribution en assistance militaire et en deniers publics, donne au peuple canadien le droit d'exprimer son avis sur la question...103

Deux députés seulement votèrent avec Bourassa une représentation du Canada aux conseils impériaux pour y déterminer les conditions de la paix qui semble aller à l'encontre de tous

100 Débats (1901), I: 1297.

101 Débats, I : 1313.

102 Débats (1901), I: 1316-1317.

103 Débats (1901), I: 1326. 
les principes de Bourassa, vigoureux adversaire de la fédération impériale. Or cette motion paraît bien exiger la fédération impériale. Toutefois si la pensée suprême de Bourassa se ramène aux principes d'un nationalisme profondément canadien, l'on peut concilier sa position à l'époque et ses déclarations antérieures. Ce qu'il veut, c'est que l'on accorde au Canada, nation autonome, fournisseuse d'hommes et d'argent dans cette guerre, quelque rôle officiel dans l'élaboration des traités qui allaient mettre fin au conflit. Cette exigence, il la présente pour maintenir le statut canadien de puissance autonome au sein de l'empire. Chamberlain, qui n'avait aucunement consulté le gouvernement canadien sur la politique de guerre, pouvait difficilement accueillir une participation réelle et active du Canada dans l'élaboration d'une politique de paix. Bourassa espérait, pour sa part, faire ressortir les intérêts divergents de la mère-partie et de la jeune nation. Il entendait prouver une fois de plus que le dessein de Chamberlain était de réduire le Canada au rang de colonie de la couronne.

Ce discours peut être considéré comme la conclusion de la lutte parlementaire de Bourassa contre l'impérialisme. Il continua de s'élever parfois contre ce qu'il croyait être des accrocs aux droits du Canada. Mais la lutte, il la transporta du Parlement à la tribune du conférencier.

En juin 1901, Bourassa partit pour l'Angleterre. Goldwin Smith l'avait muni de lettres d'introduction pour plusieurs libéraux anglais éminents, entre autres Morley, Bryce et Channing. Bourassa passa deux mois en Angleterre, n'en revenant que le 22 août. En son voyage, il s'était proposé d'aller aux sources de l'impérialisme britannique, désireux d'en accroître la connaissance. Le voyage donna lieu à une conférence donnée en français, à Montréal, le 20 octobre 1901. Imprimé en janvier de l'année suivante, le discours s'intitulait Grande-Bretagne et Canada. Il traitait de l'impérialisme, mais en son approche historique. Aux 48 pages de texte, Bourassa ajouta une préface et un appendice de 134 pages de citations extraites de documents, de débats parlementaires et de journaux, anglais et canadiens. On y sent une recherche poussée de l'auteur. La préface de 
Bourassa contient une définition, restée fameuse dans les annales canadiennes, de l'impérialisme britannique.

L'impérialisme anglais ... est un régime d'accaparement et de domination militaire, né de l'expansion exagérée de la puissance anglaise et nourri de cet orgueil stupide, brutal et vantard qu'on nomme jingoïsme. Il s'exprime volontiers par des formules ronflantes: "Britannia rules the waves...," "Britons shall never be slaves...," "Trade follows the flag...," "What we have we hold..."

Ayant embrassé plus qu'elle ne peut éteindre, entourée d'ennemis ou d'indifférents, l'Angleterre de M. Chamberlain a besoin de soldats et de marins pour maintenir les œuvres de son délire. N'en trouvant plus chez elle, elle s'adresse à ses colonies. Sachant qu'elle ne peut atteindre son but sans détours, elle trompe, endort ou corrompt les gouvernants des colonies et grise de chauvinisme leurs populations naïves. Elle nous arrache, sous des noms et des uniformes variés-Carabiniers Royaux, Infanterie à cheval, Strathcona Horse, Yeomanry tout ce qu'elle peut de chair à canon; quitte à nous laisser entrevoir quelques bribes de faveurs pour le moment où nous serons fatigués de jouer ce rôle de dupes.

En un mot, le véritable impérialisme anglais, c'est LA CONTRIBUTION DES COLONIES AUX GUERRES DE L'ANGLETERRE - en hommes et en deniers, en hommes surtout. Ce mouvement possède une caractéristique sur laquelle je voudrais attirer l'attention de mes lecteurs anglophones: je veux parler de la souple facette sous laquelle on le présente. En Angleterre, le contribuable n'entend parler que des immenses bénéfices que la mèrepatrie va en retirer: les colonies vont aider son armée et sa marine, non seulement en temps de nécessité, mais comme une institution militaire permanente qui lui épargnera l'horreur de la conscription. Aux colonies, on nous a dit que nos sacrifices libres et volontiers pour la cause impériale en Afrique du Sud devraient nous apporter d'incalculables avantages: commerce, industrie, immigration en provenance des îles britanniques, etc. 
Au Canada, politiciens de toute nuance menèrent le même double-jeu. Dans les provinces anglaises, les deux partis surenchérirent pour obtenir le prix de "loyauté" - chacun réclamant le crédit d'avoir le plus fait pour l'empire. Pas un mot de dévotion aux seuls intérêts canadiens. C'est avec terreur que les tories ont rejeté le mot fameux de leur plus grand chef: "Tant pis pour les liens britanniques!" tandis qu'aucun libéral n'oserait répéter ce que disait, en 1891, M. Laurier: "Je préfère le dollar américain au shilling anglais." Le seul point en litige, entre les deux partis, était de savoir qui mangerait la plus grosse portion du gâteau jingo... $\mathrm{Au}$ Québec, les mêmes comédiens sont apparus avec différentes perruques et différents costumes. Comme le Maître Jacques de Molière, au moment de franchir la frontière de la province, ils troquent la livrée du cocher pour le tablier du marmiton. Il n'est plus du tout question de savoir quel parti a fait le plus pour l'Angleterre, mais de savoir qui a fait le moins. Ce double jeu ne peut plus durer longtemps - mais le plus tôt il cessera, le mieux ce sera pour le Canada, la Grande-Bretagne et l'empire. Ici, il amène fatalement acrimonie et fossé entre deux races conduites comme elles le sont, par les mêmes hommes, dans deux directions opposées. Les liens britanniques, eux, seront sérieusement menacés dès que les peuples de Grande-Bretagne et des colonies découvriront les espoirs fallacieux et contradictoires qu'on leur a inculqués.

...Si j'étais l'anglophone déloyal que je suis supposé être, je demeurerais coi et heureux. Quelques années encore de ce conflit et le fossé racial se creuserait au point de nous amener, péniblement mais sûrement, vers l'annexion aux Etats-Unis. ${ }^{104}$

Le libéralisme de Bourassa encourageait une discussion ouverte et libre des problèmes. Il exécrait la duplicité qu'il retrouvait à deux niveaux: niveau impérial avec Chamberlain et ses acolytes, niveau national avec les chefs des deux partis. Duplicité et inconsistance qui contrastent radicalement avec le nationalisme à triple principe de Bourassa: affirmation de la

104 Henri Bourassa, Grande-Bretagne et Canada (Montréal, 1902), 4-5. 
liberté de l'individu vis-à-vis le parti, affirmation de l'autonomie des provinces dans le champ de la politique nationale, affirmation du nationalisme canadien au sein de l'empire. Remarquable cohérence, d'ailleurs basée sur la théorie de la décentralisation des pouvoirs à la racine même du libéralisme anglais. Dans Grande-Bretagne et Canada, Bourassa fonde là-dessus la grandeur de l'Angleterre:

L'empire britannique a été bâti d'après ce principe de décentralisation - un principe relativement nouveau, essentiellement opposé au paternalisme des empires coloniaux de l'Espagne, du Portugal, de la France... Alors, il n'est pas paradoxal de déclarer que l'empire britannique est né du sentiment antiimpérialiste du peuple anglais et que le secret de son maintien réside dans la force de cette inclination. ${ }^{105}$

Et il continue:

Il est un point théorique sur lequel $j$ 'abonde dans le sens des impérialistes doctrinaires: c'est que l'empire anglais comme toutes les institutions humaines, ne peut rester stationnaire. Il doit marcher dans la voie que lui trace l'atavisme de sa naissance et de son développement. Aux yeux de l'école libérale, la conséquence inévitable de ses prémisses est la disjonction naturelle et harmonieuse de ses parties composantes. ${ }^{106}$

Ainsi qu'il l'avait déclaré au parlement, Bourassa n'est pas opposé à tout changement dans les relations avec l'empire. Il demande simplement que les changements soient faits sur des points bien définis, discutés et décidés dans les parlements des nations concernées en ces matières. En attendant l'impérialisme rend inévitables les contributions militaires des colonies:

... où [la Grande-Bretagne] trouvera-t-elle les matériaux nécessaires pour combler ces vides? Elle n'a que deux ressources à sa disposition : la conscription ou l'appel aux colonies, - deux moyens également dangereux dans leur application. La conscrip-

105 Henri Bourassa, Grande-Bretagne et Canada (Montréal, 1902), 15. 106 Bourassa, Grande-Bretagne et Canada, 21 . 
tion, c'est le danger à l'intérieur, c'est la colère et peut-être la révolte de cinq millions d'ouvriers anglais, à greffer sur la haine de l'Irlande ;... L'appel aux colonies n'est guère plus rassurant. De contributions forcées d'hommes et d'argent, il n'en saurait être question. Mais les impérialistes se raccrochent à l'espoir qu'ont fait naître en eux la naïveté, l'enthousiasme et la facilité à se laisser duper, dont les colonies ont fait preuve depuis deux ans. ${ }^{107}$

Quelle similitude entre l'actuelle situation et l'histoire antérieure de l'Angleterre:

En somme, sous des dehors différents et avec d'autres moyens d'action, le mouvement impérialiste part du même fond et tend au même résultat que la lutte entreprise au XVIII ${ }^{\mathrm{e}}$ siècle par le gouvernement autocratique de Georges III: TAXER LES COLONIES POUR LA DEFENSE DE L'EMPIRE. Avec cette franchise qui caractérise les hommes publics d'Ángleterre et qui rend la discussion de la politique anglaise toujours intéressante et fructueuse, lord Selborne, sous-secrétaire d'Etat pour les colonies, l'a reconnu publiquement l'an dernier. La seule différence qu'il y voit, c'est que les ministres de Georges III eurent la maladresse de vouloir prélever eux-mêmes des impôts sur les colonies, tandis que les ministres actuels s'efforcent d'amener les colons à se taxer eux-mêmes au bénéfice de la métropole. ${ }^{108}$ Car c'est là, [contribution militaire coloniale] n'en doutons pas, la base du système: tout le reste, réciprocité commerciale, représentation politique, appel judiciaire, ne sont que les accessoires destinés à faire accepter le principe et en assurer le développement et la stabilité. ${ }^{109}$

Chamberlain, du reste, l'admettait ouvertement: les contributions des colonies à l'armée britannique n'étaient pas importantes en elles-mêmes, mais tiraient leur valeur du précédent qu'elles créaient pour l'avenir.

Nous apprécions leur assistance, non pas tellement à cause de son importance matérielle, même si nous

107 Bourassa, Grande-Bretagne et Canada, 27.

108 Bourassa, Grande-Bretagne et Canada, 29.

109 Loc. cit. 
la tenons, sur ce point, pour bienvenue; mais nous l'apprécions surtout parce qu'elle démontre, à la face du monde, l'unité essentielle de l'empire britannique. A l'avenir, à tout prix, que les autres autant que nos citoyens sachent bien que si une partie de l'empire est lésée, toutes les autres parties sympathiseront avec elle et l'assisteront. ${ }^{110}$

En sa brochure, Bourassa inséra, en forme d'appendices, plusieurs extraits de discours prononcés par des personnages comme lord Lansdowne et lord Wolseley. Ces extraits renforçaient sa thèse. Une déclaration explicite de son nationalisme canadien, déjà implicitement exprimée dans son opposition à la motion de sympathie d'août 1899 , conclut la brochure de Bourassa :

... il n'y a qu'un moyen possible d'éviter des malentendus funestes, c'est que nous soyons et que nous restions tous deux exclusivement Canadiens sur le terrain constitutionnel et politique. ${ }^{111}$

Le discours, nettement de ton anti-impérialiste, n'en contenait pas moins de nombreux éloges des institutions, du libéralisme et des hommes d'Etat britanniques. Caractère nullement négligeable, car le discours s'adressait à un auditoire canadienfrançais porté à confondre impérialisme et tradition britannique pour mieux les opposer l'un à l'autre. Bourassa n'a jamais voulu rompre avec les institutions britanniques ou les traditions libérales: il les admirait trop pour en venir là. Il croyait le nationalisme préconisé par lui seule expression raisonnable des traditions britanniques, dans les circonstances. Bourassa prononce un autre discours-choc, avant le départ de Laurier, en juin, pour la conférence coloniale. Le patriotisme canadienfrançais est du 27 avril 1902. Prononcé en français comme l'avait été Grande-Bretagne et Canada, le discours s'adressait spécialement aux Canadiens français. Publié plus tard, nous ne sachons pas qu'il existe une traduction anglaise. Mason Wade considère ce discours comme l'ultime effort de Bourassa pour renforcer la résistance croissante de Laurier à l'impérialisme.

110 Bourassa, Grande-Bretagne et Canada, (appendice), XL.

111 Bourassa, op. cit., 40. 
La structure du discours est la même que celle de Grande-Bretagne et Canada. Ce qu'il importe de retenir de ce discours destiné à un auditoire français, et ce, à un moment où le sentiment anti-britannique atteignait un sommet et où le nationalisme canadien-français d'allure séparatiste allait se développant, c'est que Bourassa, tout en dénonçant l'impérialisme, met encore l'accent sur son admiration pour le libéralisme et les institutions britanniques. Il décrit ainsi son attitude envers l'Angleterre.

En somme, faite sans bassesse et sans passion, cette revue historique nous amènera à la conclusion que nous ne devons à l'Angleterre ni rancune ni reconnaissance...

Notre fidélité à l'Angleterre ne peut et ne doit être qu'une affaire de raison. ${ }^{112}$

Il y aborde les problèmes de race et de nationalité.

Le premier problème quìi se pose à notre esprit, e'est celui-ci: devons-nous être plus Français que Canadiens ou plus Canadiens que Français? En d'autres termes, devons-nous être des Français au Canada ou des Canadiens d'origine française?

Je ne conçois pas qu'on puisse hésiter un instant à répondre que nous devons rester essentiellement Canadiens. ${ }^{113}$

Bourassa confesse la profondeur de son affection et de son attachement à la langue et à la culture françaises. Mais cet attachement ne doit pas incliner aux préjugés anti-anglais et il dénonce les politiciens qui recourent à de semblables appels.

C'est ce genre de patriotisme que le docteur Johnson, si je ne me trompe, a si bien caractérisé en disant qu'il est le dernier refuge des vauriens. ${ }^{114}$

A la conférence coloniale de 1902, Laurier maintient ferme sa position contre toute pression impérialiste plus accentuée.

Avant la conférence, il refusa la pairie et pendant son séjour en Angleterre, il ne se laissa aller à

112 Henri Bourassa, Le Patriotisme canadien-français, 7.

113 Bourassa, Le Patriotisme canadien-français, 10.

114 Bourassa, Le Patriotisme canadien-français, 14. 
aucun des excès d'éloquence qu'avaient caractérisé ses affirmations de 1897, pour l'embarrasser par la suite... Au National Liberal Club [Londres], il proclama: "La délégation britannique du pouvoir législatif a été le lien qui assura l'union de l'Empire britannique." 115

Juger le rôle que l'opposition de Bourassa aura pu jouer dans le changement d'attitude de Laurier qui, selon le mot même de Bourassa, passera "d'un impérialisme modéré à un nationalisme mitigé", n'est pas chose possible. ${ }^{116}$

Le facteur décisif de l'abandon, par Laurier, du principe de centralisation impériale qu'il avait paru soutenir fut le progrès du mouvement nationaliste qui faisait tache d'huile dans sa province de Québec, forteresse libérale qui lui assurait la majorité à la Chambre. ${ }^{117}$

Même avec Bourassa à la tête du mouvement nationaliste, anti-impérialisme et nationalisme dépassèrent bientôt, au Québec, l'étape de l'opposition à la politique de Chamberlain. On s'écarta de toutes les traditions anglaises pour se confiner au particularisme français. Bourassa avait insisté sur la dualité canadienne et le libéralisme britannique: le Canada français n'en tint nul compte. Il méconnut la pensée de Bourassa de la même façon, à peu de chose près, que l'avaient fait les Canadiens anglais. De son temps, Bourassa parut se faire, à certaines heures, le prophète du particularisme canadien-français, non d'un plus grand nationalisme canadien. Néanmoins ce qui fait sa grandeur, c'est qu'il n'appuya pas son opposition à l'impérialisme sur un particularisme étroit, ce qui aurait été très normal; il l'appuya sur l'affirmation d'un nationalisme canadien plus large basé sur les traditions du libéralisme anglais. Bourassa ne condescendit jamais à se départir du principe libéral d'une discussion ouverte et raisonnable pour faire appel aux sentiments et aux préjugés. Son opposition à l'impérialisme est demeurée cohérente, calme et soigneusement documentée. Ses

115 Masson Wade, The French Canadians, I: 534.

116 Bourassa, troisième Conférence, 68.

117 Wade, The French Canadians, I: 539. 
résolutions au parlement étaient des déclarations normales de la primauté des intérêts nationaux du Canada. A chaque occasion, il tenta de sauvegarder le principe de l'autonomie nationale. Bourassa le reconnaissait: des changements s'opéraient au sein de l'Empire, il les voyait croître, se développer. Interprète libéral de l'histoire de l'Angleterre, il attendait une évolution vers une plus grande autonomie canadienne. L'impérialisme lui paraissait réactionnaire, exigeant l'accroissement du sacrifice des intérêts canadiens à ceux des hommes d'État britanniques.

A ce moment de l'histoire du Canada, Bourassa seul eut le courage de sacrifier des avantages immédiats, une carrière qui s'annonçait brillante au sein du parti libéral et sous l'aile protectrice du premier ministre; il fit ce sacrifice au nom de principes qu'il croyait de prime importance pour tout le Canada. Il replaça les problèmes dans leur conjoncture historique; il éleva la politique au-dessus du jingoïsme hystérique pour la placer sur le palier de l'intérêt national raisonné.

L'étonnant, en tout cela, est que Bourassa, Canadien français cultivé, ait pu fonder son opposition à l'impérialisme sur des principes et des traditions britanniques. Son admiration du libéralisme anglais lui a permis, dans ces années difficiles, de développer ses idées d'un nationalisme canadien complet.

JAMES I. W. CORCORAN

(Traduction de Marc La Terreur)

Notes du traducteur:

1 - Lorsque les titres d'ouvrages sont indiqués en français, l'on peut conclure que la traduction donnée vient de cette version française. Ainsi, lorsque le mot Débats est employé, la traduction est celle des Débats de la Chambre des Communes.

2 - Le traducteur s'est scrupuleusement efforcé de respecter la façon dont l'auteur indique ses sources: il a poussé le scrupule jusqu'à ne pas corriger des erreurs qu'il croyait manifestes. 